\title{
Three-dimensional structure of $\beta$-cell-specific zinc transporter, ZnT-8, predicted from the type 2 diabetes-associated gene variant SLC30A8 R325W
}

\author{
Rob NM Weijers
}

\begin{abstract}
Background: We examined the effects of the R325W mutation on the three-dimensional (3D) structure of the $\beta$-cellspecific $\mathrm{Zn}^{2+}$ (zinc) transporter ZnT-8.

Methods: A model of the C-terminal domain of the human ZnT-8 protein was generated by homology modeling based on the known crystal structure of the Escherichia coli (E. coli) zinc transporter YiiP at 3.8 A resolution.

Results: The homodimer ZnT-8 protein structure exists as a Y-shaped architecture with Arg325 located at the ultimate bottom of this motif at approximately $13.5 \AA$ from the transmembrane domain juncture. The C-terminal domain sequences of the human ZnT-8 protein and the E. coli zinc transporter YiiP share 12.3\% identical and 39.5\% homologous residues resulting in an overall homology of $51.8 \%$. Validation statistics of the homology model showed a reasonable quality of the model. The C-terminal domain exhibited an a $\beta \beta a \beta$ fold with Arg325 as the penultimate Nterminal residue of the a2-helix. The side chains of both Arg325 and Trp325 point away from the interface with the other monomer, whereas the $\varepsilon-\mathrm{NH}_{3}{ }^{+}$group of Arg325 is predicted to form an ionic interaction with the $\beta$-COO-group of Asp326 as well as Asp295. An amino acid alignment of the $\beta 2$-a2 C-terminal loop domain revealed a variety of neutral amino acids at position 325 of different ZnT-8 proteins.

Conclusions: Our validated homology models predict that both Arg325 and Trp325, amino acids with a helix-forming behavior, and penultimate $\mathrm{N}$-terminal residues in the a2-helix of the C-terminal domain, are shielded by the planar surface of the three cytoplasmic $\beta$-strands and hence unable to affect the sensing capacity of the $\mathrm{C}$-terminal domain. Moreover, the amino acid residue at position 325 is too far removed from the docking and transporter parts of ZnT-8 to affect their local protein conformations. These data indicate that the inherited R325W abnormality in SLC30A8 may be tolerated and results in adequate zinc transfer to the correct sites in the pancreatic islet cells and are consistent with the observation that the SLC30A8 gene variant R325W has a low predicted value for future type 2 diabetes at population-based level.
\end{abstract}

\section{Background}

This report continues our analyses of the genetic factors playing an important role in the pathogenesis of type 2 diabetes [1]. Genome-wide association studies have currently identified single nucleotide polymorphisms (SNPs) within up to 10 genes associated with an increased risk of type 2 diabetes [2-6]. Several of the SNPs identified within or near these genes are hypothesized to influence $\beta$-cell function. Previous studies of the latter genes addi-

* Correspondence: robw01@xs4all.nl

1 Teaching Hospital OLVG, Onze Lieve Vrouwe Gasthuis, Amsterdam, The Netherlands

Full list of author information is available at the end of the article tionally identified the SNP rs13266634 as a nonsynonymous SNP causing an arginine to tryptophan change at position $325(\mathrm{R} 325 \mathrm{~W})$ in the last exon of the solute carrier family 30 (zinc transporter; $\mathrm{ZnT}$ ) member 8 (SLC30A8) gene on 8q24 (Table 1). Yet, contrary to the outcomes of the above-mentioned association studies, combining the genetic variants including the SLC3OA8 gene variant R325W was recently reported to have low predicted value for future type 2 diabetes at populationbased level [7-10]. From a different point of view, we continued our analyses to have informed discussion, and studied at atomic level the impact of the R325W muta-

(c) 2010 Weijers; licensee BioMed Central Ltd. This is an Open Access article distributed under the terms of the Creative Commons AttriBioWed Central bution License (http://creativecommons.org/licenses/by/2.0), which permits unrestricted use, distribution, and reproduction in any medium, provided the original work is properly cited. 
Table 1: Overview of confirmed type 2 diabetes association results in the combined stage 1 and 2 samples for the widely replicated type 2 diabetes-associated variant SLC30A8 R325W (rs13266634).

\begin{tabular}{|c|c|c|c|c|}
\hline Study & $\begin{array}{l}\text { Total sample size; stage } 1+2 \\
\text { (number of cases/controls) }\end{array}$ & OR $(95 \% \mathrm{CI})$ & $P$-value & Ref. \\
\hline DGI & $13,781(6,529 / 7,252)$ & $1.07(1.00-1.16)$ & 0.047 & 2 \\
\hline WTCCC/UKT2D & $13,965(5,681 / 8,284)$ & $1.12(1.05-1.18)$ & $7.0 \times 10^{-5}$ & 3 \\
\hline FUSION & $4,808(2,376 / 2,432)$ & $1.18(1.09-1.29)$ & $7.0 \times 10^{-5}$ & 4 \\
\hline $\begin{array}{l}\text { DGI-WTCCC/UKT2D- FUSION (all } \\
\text { data) }\end{array}$ & $32,554(14,586 / 17,968)$ & $1.12(1.07-1.16)$ & $5.3 \times 10^{-8}$ & $2-4$ \\
\hline Icelandic case control* & $16,398(3,836 / 12,562)$ & $1.15(1.08-1.22)$ & $3.3 \times 10^{-6}$ & 5 \\
\hline France case control & $5,511(2,617 / 2,894)$ & $1.18(0.93-1.43) \dagger 1.53(1.22-1.84) \ddagger$ & $6.1 \times 10^{-9}$ & 6 \\
\hline
\end{tabular}

*Combined European ancestry groups. †Heterozygous. ‡Homozygous.

tion on ZnT-8 complete with sensor, actuator and transporter parts.

The SLC30A8 gene encodes a 369-amino acid protein, $\mathrm{ZnT}-8$, that transports $\mathrm{Zn}^{2+}$ (zinc) from the cytoplasm into insulin secretory vesicles, where insulin is stored as a hexamer bound with two zinc ions before secretion [1114]. The ZnT-8 protein is specifically expressed in pancreatic $\beta$-cells and thus may be of primary importance for the insulin secretory pathway. Variations in SLC30A8 may affect zinc accumulation in insulin granules, affecting insulin stability, storage, or secretion.

In mammalian cells, eight homologous zinc export proteins, named ZnT-1 to -8 , have been described $[11,14]$. These proteins are members of the SLC30 subfamily of the cation diffusion facilitator family. Analysis of genome sequences shows that cation diffusion facilitators represent a ubiquitous protein family, encompassing more than 400 evolutionarily related members found in species ranging from bacteria and yeast to plants and mammals [15-17]. This protein family is characterized by an N-terminal hydrophobic domain in addition to a $\mathrm{C}$-terminal cytosolic, hydrophilic region that is highly variable both in sequence and in length [18] with a common and remarkably evolutionarily conserved $\alpha \beta \beta \alpha \beta$ fold. Despite the relatively low sequence homology in the cytoplasmic domains, the structural homology between them is impressive. For example, the $\alpha \beta \beta \alpha$ structural core of the $C$-terminal domain from $\alpha 1$ to $\alpha 2$ of the $E$. coli zinc transporter YiiP can be superimposed onto the equivalent portion of human copper metallochaperone Hah1 with a root mean square deviation of $1.8 \AA$ for 42 common $C_{\alpha}$ positions, although there is no sequence homology between the C-terminal domain and Hah1 after a evolutionary period of more than thousand millions of years [[19], see ref. [21]], while the soluble fragment from Thermus thermophilus zinc transporter CzrB overlays the cytoplasmic domain of YiiP with a $\mathrm{C}_{\alpha}$-root mean square deviation of $1.8 \AA$ over 79 residues with an overall sequence identity of $8.8 \%$ [20].

The zinc transporting function is attributed to the homologous hydrophobic domain, which is thought to be composed of a bundle of six transmembrane segments, denoted as TM1 to TM6, in an $\alpha$-helical configuration, a structural theme found in many other membrane channels and transporters [21,22]. A representative member of the cation diffusion facilitator family is YiiP, a dimer of two identical 33-kD integral zinc transporter membrane proteins found in the plasma membrane of E. coli [23]. Recently, X-ray diffraction analysis of YiiP crystals and 12 heavy-atom derivative crystals revealed YiiP dimers at a resolution of $3.8 \AA$ with individual subunits, each containing six bilayer-spanning $\alpha$-helices, a C-terminal domain with an $\alpha \beta \beta \alpha \beta$ fold, and four zinc-populated sites (Fig. 1) [19,24]. Most recently, the C-terminal domain of CzrB was overexpressed in E. coli and the crystal structure of the apo and zinc forms of the soluble fragment $\left(\mathrm{CzrB}_{\mathrm{sf}}\right)$ solved to 1.7 and $1.8 \AA$, respectively [20]. We have used this, in combination with the structure of YiiP, to examine the effects of the missense mutation on the 3D structure of the ZnT-8 protein complete with sensor, actuator and transporter parts.

\section{Methods}

We retrieved information regarding the amino acid sequences of human ZnT-2 (Q9BRI3), ZnT-3 (Q99726), and ZnT-8 (Q8IWU4), mouse ZnT-8 (Q8BGG0), E. coli zinc transporters YiiP (Q1R404) and ZitB (Q8×400), Thermus thermophilus zinc transporter CzrB (Q8vlX7) and Ralstonia metallidurans zinc transporter $\mathrm{CzcD}$ (P13512) from the UniProtKB/Swiss-Prot databank [25]. The crystal structure of YiiP was retrieved from the Protein Data Bank [19,26]. C-terminal domains of Homo 


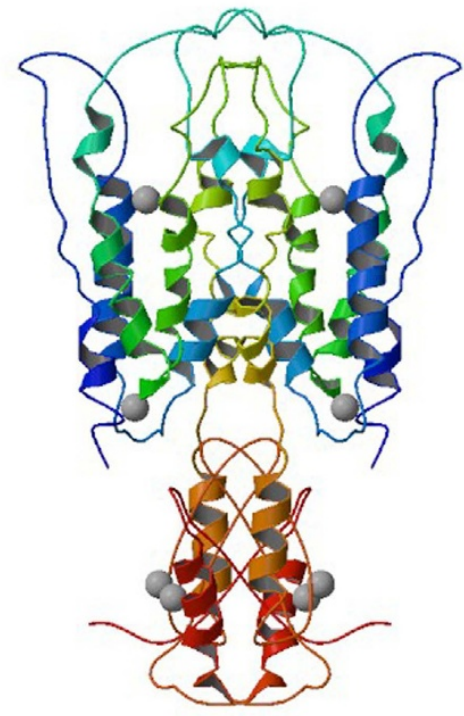

Figure 1 Biological molecule image for the zinc transporter YiiP (17). The biological molecule consists of a pair of identical protein chains, each composed of (a) six transmembrane domains (blue and green) at the extracellular side, (b) a hydrophilic C-terminal domain with a $a \beta \beta a \beta$ fold (red and orange) at the intracellular side, and (c) four zinc-populated sites (top-down designated as Z1 to Z4). Zinc ions are represented by gray spheres.

sapiens $\mathrm{ZnT}-8$ and E. coli zinc transporter YiiP sequences were aligned and with a typical homology modeling exercise, the most probable 3D models were created with the program Modeller, version 9 [27]. The model was validated using the PSVS validation server [28-31] exclusive of the MolProbity clashscore because for this sort of analysis to be reliable, the reference crystal structure needs to be better than $2.0 \AA$ resolution [32] (Table 2). All model representations were prepared using the program Pymol (DeLano Scientific) [33]. Secondary structure elements of the $\mathrm{C}$-terminal domain of the zinc transporters $\mathrm{CzrB}$ were indicated as $\alpha$-helices and $\beta$-pleated sheets according to the specifications of Cherezov et al. [20].

\section{Results}

We focused our analyses on the C-terminal domain of ZnT-8 because position 325, at which the mutation takes place, is located on this part of the protein. A model of the C-terminal domain of the human ZnT-8 protein was generated by homology modeling based on the known crystal structure of the $E$. coli zinc transporter YiiP at 3.8 $\AA$ resolution. The two sequences share $12.3 \%$ identical and 39.5\% homologous residues (Fig. 2) resulting in an overall homology of $51.8 \%$, which is well within the safe limits for homology modeling. A model was generated with Modeller [27] and validated using the PSVP validation server (Table 2) [28]. The generated models of the
Table 2: Validation statistics of the homology model of the C-terminal domain of YiiP generated with the PSVS server .

Structure quality factors - overall statistics

\begin{tabular}{lll}
\hline & Mean score & Z-scorea \\
Procheck G-factor (phi/psi only) [29] & -0.75 & -2.64 \\
Procheck G-factor (all dihedral angles) & -0.49 & -2.90 \\
[29] & & \\
Verify3D [30] & 0.22 & -3.85 \\
Prosall (-ve) [31] & 0.09 & -2.32 \\
Ramachandran plot summary from Procheck [29] & \\
Most favoured regions & $72.0 \%$ & \\
Additionally allowed regions & $17.3 \%$ & \\
Generously allowed regions & $8.0 \%$ & \\
Disallowed regions & $2.7 \%$ & \\
\hline
\end{tabular}

aWith respect to mean and standard deviation for a set of $252 \mathrm{X}$ ray structures $<500$ residues, of resolution $\leq 1.80 \AA$, $R$-factor $\leq$ 0.25 and $\mathrm{R}$-free $\leq 0.28$; a positive value indicates a 'better' score.

wild-type ZnT-8 protein and its Arg325Trp variant revealed a Y-shaped architecture consisting of a pair of identical protein chains, each composed of six transmembrane domains, a hydrophilic C-terminal domain with a $\alpha \beta \beta \alpha \beta$ fold and four zinc receiving domains (designated as Z1 to Z4) (figure not shown). In our model, the Arg325 side-chain is at approximately $15 \AA$ from the $\mathrm{Z} 2$ site.

Fig. 3 displays the superposition of the loop connecting the second $\beta$-strand to the second $\alpha$-helix of the $\mathrm{C}$-terminal domain of the ZnT-8 R325W variant upon the structure of the YiiP zinc transporter. The $\alpha 2$-helix of the ZnT-8 mutated protein has Trp325 as the penultimate $\mathrm{N}$-terminal residue. Further, the side-chain of $\operatorname{Trp} 325$ points away from the interface with the other monomer. In the wildtype ZnT- 8 protein, the $\alpha 2$-helix has Arg325 as the penultimate $\mathrm{N}$-terminal residue, while the Arg325 side-chain has an orientation virtually identical to that of Trp325 in the ZnT-8 R325W variant (Fig. 4). Arg325 may form an interchain noncovalent, electrostatic interaction (salt bridge) with its adjacent Asp326 and an intrachain salt bridge with Asp295 of the $\alpha 1$-helix of the C-terminal domain (Fig. 4). Table 3 shows the alignment of loop-domains linking the second $\beta$-strand to the second $\alpha$-helix of the $C$-terminal domain of ZnT-8 and other ZnT proteins. The secondary structure elements ( $\alpha$-helix, $\beta$-strand, and loop) are indicated according to the 1.8 Â resolution zinc- $\mathrm{CzrB}_{\mathrm{sf}}$ model [20]. Position 325, at which the mutation takes place, corresponds to a charged Arg residue. By replacing it with a Trp residue, the positive charge is removed, thereby affecting the electrostatic interactions with the protein. 


\section{ALPDE-ERQEIIDIVTSWPGVSGAHDLRTR-QSGPTRFIQIHLEMEDSLPLVQAHMVADQ

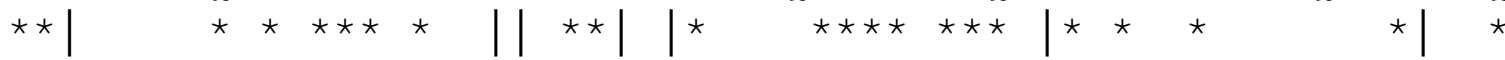
277 GVPKSLNYSGVKELILAVDGVLSVHSLHIWSLTMNQVILSAHV-ATAA--SRDSQVVRRE

\section{VEQAILRRFPGSDVIIHQDPCSVV

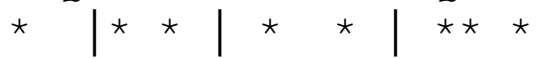

\section{IAKALSKSFTMHSLTIQMESPVDQ}

Figure 2 Alignment of the human ZnT-8 (lower row) and E. coli zinc transporter YiiP (upper row) C-terminal domain sequences. Amino acid residues are indicated by single letters. A vertical line connects identical residues and $a *$ connects homologous residues.

\section{Discussion}

This report is a description of a $3 \mathrm{D}$ atomic-resolution model of the C-terminal domain of a $\beta$-cell-specific $\mathrm{Zn}^{2+}$ transporter, ZnT-8, predicted from the type 2 diabetesassociated gene variant SLC30A8 R325W. As for studies in zinc transporter functioning [20,34], we used homology modeling, which is based on the general observation that evolutionarily related (homologous) proteins are likely to have similar structures and currently gives the most accurate and reliable models [35]. We built structural models for ZnT-8 and its gene variant R325W, based on the known structure of a close homologue (template) YiiP, a membrane transporter that catalyzes $\mathrm{Zn}^{2+} / \mathrm{H}^{+}$ exchange across the inner membrane of E. coli. The qual-

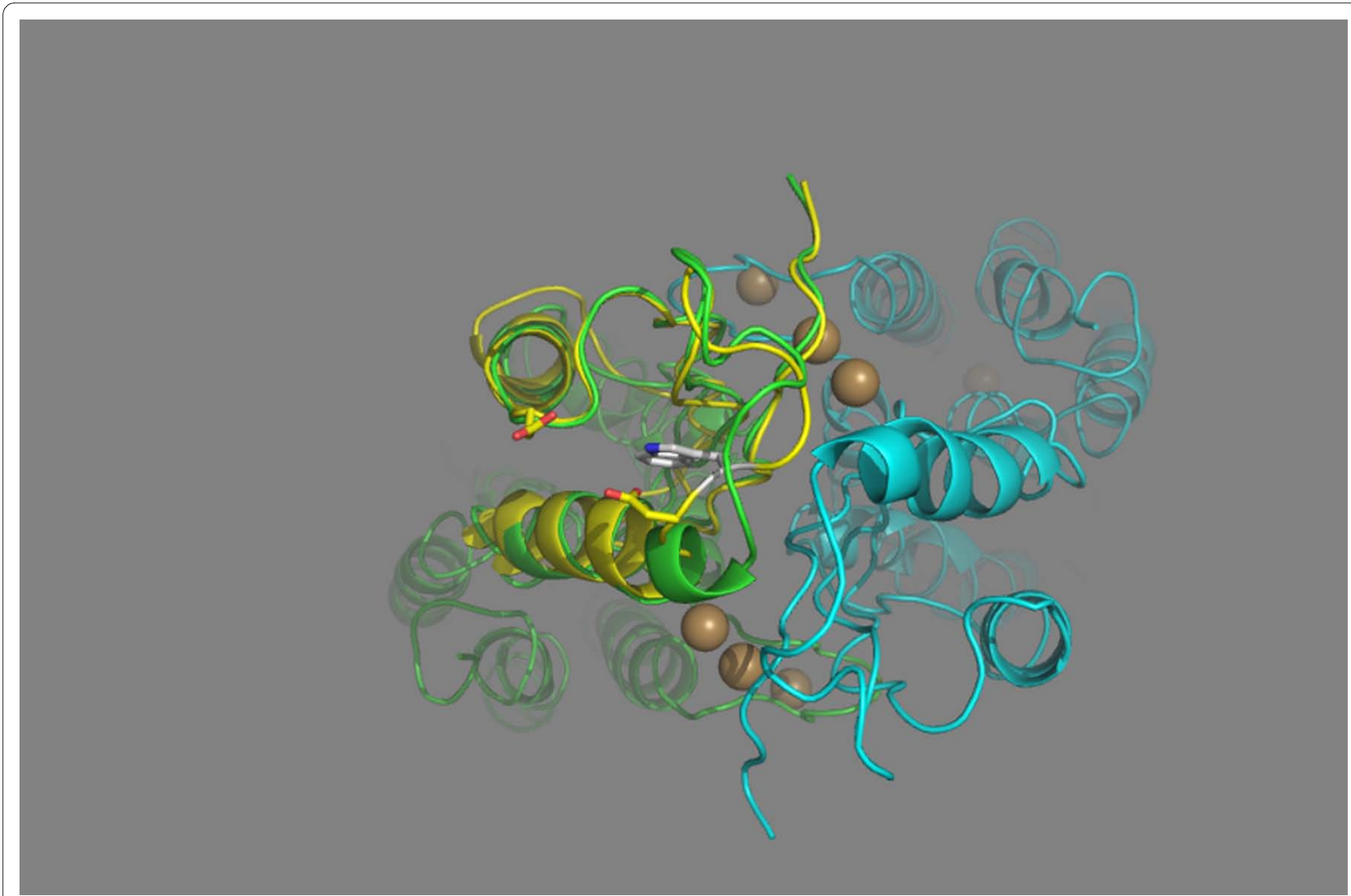

Figure 3 In this diagram of the ZnT-8 R325W variant, the loop domain linking $\beta 2$ to a2 at the outer edge of the C-terminal domain intracellular part faces the viewer. Note that Trp325 points away from the interface with the other monomer. The bacterial structure is indicated in green, the human structure in yellow, and the other monomer in blue. The four front spheres at the interface of the intracellular domains represent the positioning of the two binuclear zinc ion clusters in sites Z3 and Z4 in each of the protomers, and the faint back spheres the zinc ion in site Z2 in each of the protomers. The side-chains of Trp325, Asp326, and Asp295 are presented by a "ribbon structure". 


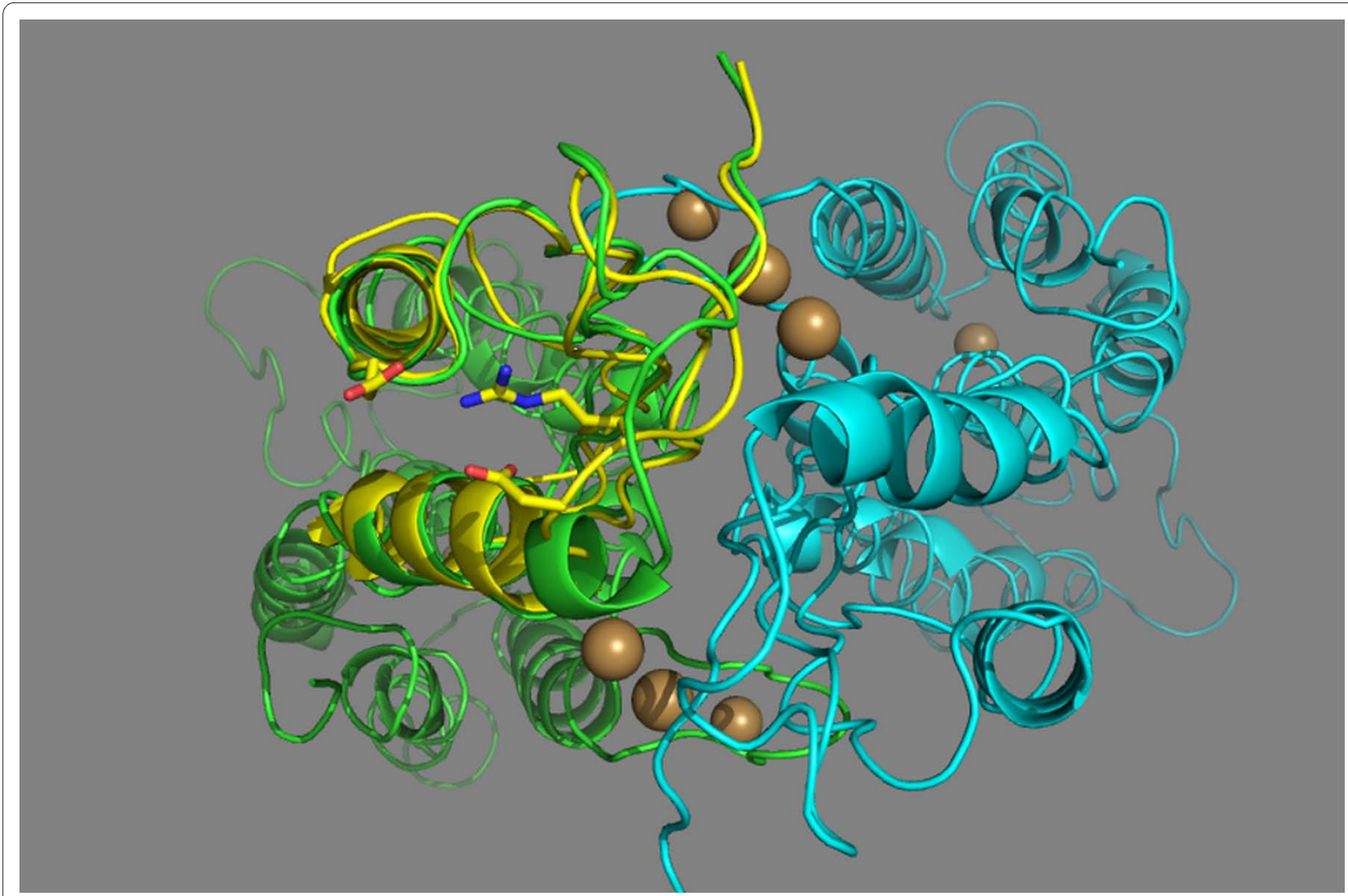

Figure 4 Close-up view of the loop domain linking $\beta 2$ to $\alpha 2$ (in front of the figure) at the outer edge of the C-terminal domain intracellular part of the 3D structure of the wild-type ZnT-8 protein. Note that Arg325 points away from the interface with the other monomer. The bacterial structure is indicated in green, the human structure in yellow, and the other monomer in blue. The four front spheres at the interface of the intracellular domains represent the positioning of the two binuclear zinc ion clusters in sites Z3 and Z4 in each of the protomers, and the back spheres the zinc ion in site $\mathrm{Z} 2$ in each of the protomers. At the back of the figure, the faint sphere represents a zinc ion in site Z1. The model predicts salt bridges linking the $\varepsilon-\mathrm{NH}_{3}{ }^{+}$group of Arg325 and the $\beta$-COO- group of Asp326 as well as Asp295. The side-chains of Arg325, Asp326, and Asp295 are presented by a "ribbon structure".

\section{Table 3: Sequence alignment of the loop domain linking $\beta 2$ to $\alpha 2$ of YiiP and representative members of the cation} diffusion facilitator family.

\begin{tabular}{lll}
\hline CDF homologs & Amino acid sequence & Residue number \\
\hline YiiP & PTRFIQIHLE MEDSLP LVQAHMVADQVEQAI \\
CzrB & PRSFLEFHLV VRGDTP VEEAHRLCDELERAL \\
ZitB & EKPVMTLHVQ VI---P PHDHDALLDQIQHYL & 286 \\
CzCD & GKASLTVHVV NDTA-- VNPEMEVLPELKQML & 269 \\
msZnT-8 & NQVILSVHVA TA---A SQDSQSVRTGIAQAL \\
hmZnT-2 & AQPVLSVHIA IAQN-- -TDAQAVLKTASSRL \\
hmZnT-3 & TYHVASAHLA IDST-- -ADPEAVLAEASSRL \\
hmZnT-8 & NQVILSAHVA TA---A SRDSQVVRREIAKAL & 337 \\
\end{tabular}

The human ZnT-8 residue R325 is in boldface. YiiP and ZitB are bacterial CDF homologs from Escherichia coli, CzcD from Ralstonia metallidurans, CzrB from Thermus thermophilus, msZnT-8 from Mus musculus (mouse), and ZnT-2, ZnT-3, and ZnT-8 from Homo Sapiens. 
ity of the homology model is dependent of the quality of the sequence alignment and template structure [36]. Alignment of the ZnT-8 and YiiP C-terminal domain sequences revealed an overall homology of $51.8 \%$, which is well within the safe limits for homology modeling (Fig. 2). The model had $89.3 \%$ residues in the allowed regions of the Ramachandran plot, a value consistent with a value of 90.1\% reported for the total structure of ZnT-8 [37].

The YiiP protein shows the main characteristics of a family of ubiquitous zinc transporters termed cation diffusion facilitator including: (a) a homodimer held together in a parallel orientation through four zinc ions at the interface of the cytoplasmic domains, with the two transmembrane domains swung out to yield a Y-shaped structure; (b) a 33-kD integral transmembrane protein composed of a compact bundle of six transmembrane segments in an $\alpha$-helical configuration (residues 1-211) arranged in a way that creates an extra- and an intracellular cavity on either side of the membrane to make room for one zinc ion, Z1 and Z2, respectively; and, finally, (c) a hydrophilic C-terminal domain (residues 212-300) consisting of three $\beta$-strands and two $\alpha$-helices ( $\alpha 1-\beta 1-\beta 2-$ $\alpha 2-\beta 3$ order). The latter exists as a three-stranded antiparallel $\beta$-sheet with the overall shape of a flattened ellipsoid. The rounded part of the domain is made up of the two $\alpha$-helices laying on top of the $\beta$-sheets [38-40]. At the interface with the other monomer and close to the $\beta$ sheets, a binuclear zinc cluster is located at the sites Z3 and Z4 (Figs. 3 and 4). Finally, the ZnT- 8 protein was predicted to adopt the same topology as the other $\mathrm{ZnT}$ proteins with six transmembrane helices [11]. Collectively, the data presented here suggest that the quality of the sequence alignment and template structure tend to be reliable for producing a 3D model of the ZnT-8 C-terminal domain to investigate the structural consequences of the substitution of Trp for Arg at position 325.

Recently, Cherezov et al. reported seminal data on the mechanism of transmembrane zinc transport via the putative zinc transporter CzrB [20]. Briefly, the proposed transport model suggests that in the absence of zinc the two flattened ellipsoids $(\alpha \beta \beta \alpha \beta$ motif) as a dimer are splayed apart by charge repulsion. This will contribute to holding the two $\mathrm{C}$-terminal domains apart and will serve to direct zinc ions into Z3 and Z4 as the intracellular concentrations of the ion rise. Thus, the cytosolic zinc concentration is sensed by the exposed C-terminal domain. When zinc binds to Z3 and Z4, a large conformational change occurs that closes the two ellipsoids $(\alpha \beta \beta \alpha \beta$ motif) and hence enlarge the dimerization surface area from $\sim 400 \AA$ in the absence of zinc to $\sim 1050 \AA$ in its presence. The net effect of this zinc binding is to change the relative disposition of the cytosolic Z2 domain environment in such a way that a zinc-loaded metallochaperone can dock for delivery and transport of its zinc cargo to this intracellular cavity. The ion then travelers across the membrane into the extracellular cavity Z1 where effectively it is out of the cell.

Our homology model predicts that the Arg325 sidechain is located at approximately $15 \AA$ from the compact bundle of six transmembrane helices. It is thus rather distant from the transmembrane domain site including the extra- and the intracellular cavity Z1 and Z2, respectively, which suggests that the Arg to Trp substitution at position 325 is unlikely to affect the protein folding near the cavity loci and the uploading of zinc.

The overall shape of the C-terminal domain is that of an flattened ellipsoid existing of three $\beta$-strands with the central strand running parallel and anti-parallel to its neighbor on either side and on top two $\alpha$-helices. The three $\beta$-sheets create a planar and distinctly polar surface of the ellipsoid with a pronounced negative charge at the interface with the other monomer, the region with the bridged zinc ions Z3 and Z4 [20]. Our models predict that both the side chain of Arg325 and Trp325 point away from the interface with the other monomer (Fig. 3, 4). These data suggest that the amino acid residue at position 325 is neither involved in dimerization contacts, nor in the zinc coordination sites $\mathrm{Z} 3$ and $\mathrm{Z} 4$, and that the three $\beta$-sheets shield the positive charge of the $\varepsilon-\mathrm{NH}_{3}{ }^{+}$group of Arg325 from the sites Z3 and Z4 [[19], see Fig. S3; [20], see Fig. 3]. The existence of an positively charged Arg325 side chain on the outside of the ellipsoid pointing away from the interface with the other monomer in the presence a pronounced negative charge on the inner side of the ellipsoid reflects this effective way of shielding. The above data support the concept that the substitution of Trp for Arg at position 325 is unlikely to affect the protein folding of the exposed C-terminal domain, the sensor of the cytosolic zinc concentration.

Furthermore, our model of the wild-type ZnT-8 predicts an inter- and an intrachain salt bridge linking the $\varepsilon$ $\mathrm{NH}_{3}{ }^{+}$group of Arg325 to both the $\beta-\mathrm{COO}^{-}$group of Asp326 and Asp295. Because the magnitude of the electrostatic force between two point electric charges is inversely proportional to the square of the total distance between the two charges, the magnitude of the electrostatic force between Arg325 and Asp295 is approximately $25 \%$ compared to the magnitude of the electrostatic force between Arg325 and Asp326. These observations suggest that the electrostatic interactions marginally contribute to the stabilization of the $\alpha \beta \beta \alpha \beta$ fold of the C-terminal domain. A Trp325 would fit there, but the salt bridges would be lost, and this loss could alter the conformational state of the $\alpha 1$-helix and, in turn, the zinc binding and transport. Surprisingly, sequence alignment of wild-type ZnT-8 and YiiP revealed that Arg325 found in the former protein seems to be replaced by a Val residue in the latter 
protein, implying the disappearance of the salt bridges in the protein structure of YiiP (Table 2). For similar reasons, these salt bridges are absent in the prototypes of the bacterial members of the cation diffusion facilitator family homologs CzrB from Thermus thermophilus, ZitB from E.Coli and $\mathrm{CzcD}$ from Ralstonia metallidurans, and in mouse ZnT-8 (Table 2). A sequence alignment of the C-terminal domain from the ZnT-2, -3, and -8 proteins revealed that Arg325 in ZnT-8 was substituted by Thr279 in ZnT-2 and by Ala343 in ZnT-3 (Table 2). Additionally, an analysis of the phylogenetic tree for all identified human ZnT family proteins indicated that ZnT-2, -3, and -8 cluster closely together [19]. Thus, independent of the existence of the inter- and intrachain salt bridges, all currently known structures of metallochaperones and their target domains fully control the activity of intracellular zinc transport [39].

In contrast to our study on ZnT-8, Nicolson et al. reported that cells expressing the ZnT-8 Trp325 protein transport more zinc into cells, than cells expressing wildtype ZnT-8 Arg325 protein [37]. However, these observations are likely incorrect, because these conclusions appear to be based on an assay for ZnT-8 transporter activity revealing that the transporter may operate in the reverse direction whereas there are no ATPase domains in ZnT-8 which may be required for ZnT-8 to operate in the reverse direction. For instance, non-specific events may be responsible for the observed different rates of zinc transport, i.e., difference in the number of zinc transporters per area unit of secretory granules of $\beta$-cells expressing either wild-type or Trp325 variant of ZnT-8, or difference in background color of microscopy images of control cells versus $\beta$-cells expressing either wild-type or Trp325 variant of ZnT-8, as very similar experiments revealed a substantial amount of zinc in control cells in normal conditions versus $\mathrm{ZnT}$-8-expressing cells, i.e., 830 $\pm 109 \mu \mathrm{g} \mathrm{Zn} / \mathrm{g}$ protein and $1072 \pm 78 \mu \mathrm{g} \mathrm{Zn} / \mathrm{g}$ protein, respectively [12]. Nicolson et al. also concluded that the resultant introduction of positive charge into the region of monomer interface of ZnT-8, close to the predicted sites of bound structurally important zinc ions, may be expected to affect the kinetics of zinc transport [37]. Without any particular detail of a 3D model for ZnT-8, their conclusions are based merely on the observation that R325 resides at the monomer interface as our models predict that the side chains of both Arg325 and Trp325 point away from the interface with the other monomer. To resolve the above discussed differences and to show that wild-type Arg325 and Trp325 variant of ZnT-8 are no different in their ability to form dimers and their ability to transport zinc, we propose to improve the experimental conditions to prevent reverse zinc transport as described for ZnT-8 [37] and ZnT-5 [41]. Next, we propose the following sequence of experiments: (a) to assay directly the functional activity of ZnT-8, measure the transmembrane flux of zinc across the plasma membrane of Xenopus laevis oocytes [41]; (b) inject oocytes with wild-type Arg325 ZnT-8 RNA, with Trp325 variant of ZnT-8 RNA or water; (c) assay zinc uptake into oocytes by measuring ${ }^{65} \mathrm{Zn}^{2+}$ incorporation into single oocytes as $\gamma$ emission [42].

According to the data presented by Cherezov et al. [20], Arg325 is a part of the $\alpha 2$ helical structure of the C-ternminal domain (Table 2). Arg has a helix-forming behavior and its tendency is to stabilize $\alpha$-helices [43]. Also, Trp has a helix-forming behavior, which makes it unlikely that the R325W mutation affects the $\alpha 2$-helix folding.

An obvious limitation of our study is largely inherent to all theoretical models in biochemistry in general, which never allow complete certainty [36]. Our model of the ZnT-8 protein was based on a crystal structure of the $E$. coli zinc transporter YiiP. In the future, a high resolution ZnT-8 structure model will help to identify all specific residues that may contribute to full transport activity of zinc. Furthermore, we cannot fully exclude the possibility that the presence of a Trp residue at position 325 does not interfere with the binding of a putative zinc metallochaperone. However, cross-sectional analyses of 75-g 3$\mathrm{h}$ oral glucose tolerance test results for a large sample of Caucasians and for populations with an exceptionally high prevalence of type 2 diabetes revealed inverted-U plots of insulin vs. glucose in plasma, implying direct proof of excellent insulin secretion during the pre-diabetic state, and consequently of adequate zinc transfer to the correct sites in the pancreatic islet cells [44-46].

\section{Conclusions}

This modeling predicts that both Arg325 and Trp325, amino acids with a helix-forming behavior and penultimate $\mathrm{N}$-terminal residues in the $\alpha 2$-helix of the $\mathrm{C}$-terminal domain, are shielded by the planar surface of the three cytoplasmic $\beta$-strands and hence unable to affect the sensing capacity of the $\mathrm{C}$-terminal domain. Moreover, the amino acid residue at position 325 is too far removed from the docking and transporter parts to affect their local protein conformations. These data suggest that the inherited R325W abnormality in SLC30A8 may be tolerated and results in adequate zinc transfer to the correct sites in the pancreatic islet cells and provide support to assume that the $S L C 30 A 8$ gene variant R325W has a low predicted value for future type 2 diabetes at populationbased level [7-9].

\section{Abbreviations}

3D: three-dimensional; E.coli: Escherichia coli; SLC30A8: solute carrier family 30 zinc transporter member 8; SNP: single nucleotide polymorphism; ZnT: zinc transporter.

\section{Competing interests}

The author declares that they have no competing interests. 


\section{Acknowledgements}

I am grateful to Prof. A.M.J.J. Bonvin (Education Director Chemistry, NMR, Utrecht University, Utrecht, The Netherlands) for the performance of the molecular modeling experiments, and helpful discussions concerning this work.

\section{Author Details}

Teaching Hospital OLVG, Onze Lieve Vrouwe Gasthuis, Amsterdam, The Netherlands

Received: 26 January 2010 Accepted: 5 June 2010 Published: 5 June 2010

\section{References}

1. Weijers RNM: Risk loci for type 2 diabetes - Quo vadis? [minireview]. Clin Chem Lab Med 2009, 47:383-386.

2. Diabetes Genetics Initiative of Broad Institute of Harvard and MIT, Lund University, and Novartis Institutes of BioMedical Research: Genome-wide association analysis identifies loci for type 2 diabetes and triglyceride levels. Science 2007, 316:1331-1336.

3. Zeggini E, Weedon MN, Lindgren CM, Frayling TM, Elliott KS, Lango H, et al:: Replication of genome-wide association signals in UK samples reveals risk loci for type 2 diabetes. Science 2007, 316:1336-1341.

4. Scott L, Mohlke KL, Bonnycastle LL, Willer CJ, Li Y, Duren WL, et al:: A genome-wide association study of type 2 diabetes in Finns detects multiple susceptibility variants. Science 2007, 316:1341-1345.

5. Steinthorsdottir $V$, Thorleifsson G, Reynisdottir I, Benediksson R, Jonsdottir $T$, Walters GB, et al: A variant in CDKALI influences insulin response and risk of type 2 diabetes. Science 2007, 316:770-775.

6. Sladek R, Rocheleau G, Rung J, Dina C, Shen L, Serre D, et al:: A genomewide association study identifies novel risk loci for type 2 diabetes. Nat 2007, 445:828-830.

7. A Meigs JB, Shrader P, Sullivan LM, MCAteer JB, Fox CS, Dupuis J, et al:: Genome score in addition to common risk factors for prediction of type 2 diabetes. N Eng/ J Med 2008, 359:2208-2219.

8. Hoek van M, Dehghan A, Witteman JCM, Duijn van CM, Uitterlinden AG, Oostra BA, et al: Predicting type 2 diabetes based on polymorphisms from genome-wide association studies. A population-based study. Diabetes 2008, 57:3122-3128.

9. Lango H, Palmer CAN, Morris AD, Zeggini E, Hattersley AT, McCarthy MI, et al: Assessing the combined impact of 18 common genetic variants of modest effect sizes on type 2 diabetes risk. Diabetes 2008, 57:3129-3135

10. D Miyake K, Yang W, Hara K, Horikawa Y, Osawa H, et al: Construction of a prediction model for type 2 diabetes mellitus in the Japanese population based on 11 genes with strong evidence of the association. $J$ Hum genet 2009, 54:236-241.

11. Chimienti F, Devergnas S, Favier A, Seve M: Identification and cloning of a $\beta$-cell-specific zinc transporter, ZnT-8, localized into insulin secretory granules. Diabetes 2004, 53:2330-2337.

12. Chimienti F, Devergnas S, Pattou F, Schuit F, Garcia-Cuenca R, Vandewalle $B$, et al: In vivo expression and functional characterization of the zinc transporter ZnT8 in glucose-induced insulin secretion. J Cell Sci 2006, 119:4199-4206.

13. Dunn MF: Zinc-ligand interactions modulate assembly and stability of the insulin hexamer [review]. Biometals 2005, 18:295-303.

14. Chimienti F, Aouffen M, Favier A, Seve M: Zinc homeostasis-regulating proteins: new drug targets for triggering cell fate. Curr Drug Targets 2003, 4:323-328.

15. Bateman A, Coin L, Durbin R, Finn RD, Hollich V, Griffiths-Jones S, et al:: The Pfam protein families database. Nucleic Acids Res 2004, 32:138-141.

16. Haney CJ, Grass G, Franke S, Rensing C: New developments in the understanding of the cation diffusion facilitator family. I Industrial Microbiol \& Biotech 2005, 32:215-226.

17. Montanini B, Blaudez D, Jeandroz S, Sanders D, Chalot M: Phylogenetic and fuctional analysis of the Cation Diffusion Facilitator (CDF) family: improved signature and prediction of substrate specificity. $B M C$ Genomics 2007, 8:107. [PubMed: 17448255]

18. Paulsen IT, Saier MH Jr: A novel family of ubiquitous heavy metal ion transport proteins. J Membr Biol 1997, 156:99-103.

19. Lu M, Fu D: Structure of the zinc transporter YiiP. Science 2007, 317:1746-1748
20. Cherezov V, Höfer N, Szebenyl DME, Kolaj O, Wall JG, Gillilan R, et al: Insights into the mode of action of a putative zinc transporter CzrB in Thermus thermophilus. Structure 2008, 16:1378-1388.

21. Agre $\mathrm{P}$, Bonhivers $\mathrm{M}, \mathrm{Borgnia} \mathrm{MJ}$ : The aquaporins, blueprints for cellular plumbing systems. J Biol Chem 1998, 273:14659-14662.

22. Tomii K, Kanehisa M: A comparative analysis of $A B C$ transporters in complete microbial genomes. Genome Res 1998, 8:1048-1059.

23. Wei Y, Li H, Fu D: Oligomeric state of the Eschericia coli metal transporter YiiP. J Biol Chem 279, 38:39251-39259.

24. Protein Data Bank [http://www.pdb.org/pdb/explore/ explore.do? structureld=2OFl]

25. Gasteiger E, Gattiker A, Hoogland C, Ivany I, Appel RD, Bairoch A, et al: ExPASy: the proteomics server for in-depth protein knowledge and analysis. Nucleic Acids Res 2003, 31:3784-3788.

26. Berman HM, Westbrook J, Feng Z, Gilliland G, Bhat TN, Weissig H, et al:: The Protein Databank. Nucleic Acids Res 2000, 28:235-242.

27. Sali A, Blundell TL: Comparative protein modelling by satisfaction of spatial restraints. J Mol Biol 1993, 234:779-815.

28. Bhattacharya A, Tejero R, Montelione GT: Evaluating protein structures determined by structural genomics consortia. Proteins: Structure, Function, and Bioinformatics 2007, 66:778-795.

29. Laskowski RA, MacArthur MW, Moss DS, Thornton JM: PROCHECK: A program to check the stereochemical quality of protein structures. $J$ Appl Cryst 1993, 26:283-291.

30. Lüthy R, Bowie JU, Eisenberg D: Assessment of protein models with three-dimensional profiles. Nature 1992, 356:83-85.

31. Sippl MJ: Recognition of errors in three-dimensional structures of proteins. Proteins 1993, 17:355-362.

32. Word JM, Bateman RC Jr, Presley BK, Lovell SC, Richardson DC: Exploring steric constraints on protein mutations using MAGE/PROBE. Prot SCi 2000, 9:2251-2259.

33. DeLano WL: The PyMOL Molecular Grafics System Palo Alto: DeLano Scientific LLC; 2002

34. Nicolson TJ, Bellomo EA, Wijesekara N, Loder MK, Baldwin JM, Gyulkhandanyan AV, et al: Insulin storage and glucose homeostasis in mice null for the granule zinc transporter ZnT8 and studies of the type 2 diabetes-associated variants. Diabetes 2009, 58:2070-2083.

35. Chothia C, Lesk AM: The relation between the divergence of sequence and structure in proteins. EMBO J 1986, 5:823-826.

36. Tai K, Fowler P, Mokrab Y, Stansfeld P, Sansom MPS: Molecular modeling and simulation studies of ion channel structures, dynamics and mechanisms. Methods Cell Biol 2008, 90:233-265.

37. Nicolson TJ, Bellomo EA, Wijesekara N, Loder MK, Baldwin JM, Gyulkhandanyan AV, et al:: Insulin storage and glucose homeostasis in mice null for the granule zinc transporter ZnT8 and studies of the type 2 diabetes-associated variants. Diabetes 2009, 58:2070-2083.

38. Hung IH, Casareno RLB, Labesse G, Mathews FS: HAH1 is a copperbinding protein with distinct amino acid residues mediating cpper homeostasis and antioxidant defense. J Biol Chem 1998, 273:1749-1754.

39. Finney LA, O'Halloran TV: Transition metal speciation in the cell: insights from the chemistry of metal receptors. Science 2003, 300:931-936.

40. Rosenzweig AC, O'Halloran TV: Structure and chemistry of the copper chaperone proteins. Curr Opin Chem Biol 2000, 4:140-147.

41. Valentine RA, Jackson KA, Christie GR, Mathers JC, Taylor PM, Ford D: ZnT5 variant $B$ is a bidirectional zinc transporter and mediates zinc uptake in human intestinal Caco-2 cells. J Biol Chem 2007, 282:14389-14393.

42. Peter GJ, Davies A, Watt PW, Birrell J, Taylor PM: Interactions between the thiol-group reagent $\mathrm{N}$-ethylmaleimide and neutral and basic amino acid transporter-related amino acid transport. Biochem J 1999, 343:169-176.

43. Garrett RH, Grisham CM: Biochemistry 2nd edition. Fort Worth: Saunders College Publishing; 1999.

44. Yki-Järvinen $\mathrm{H}$ : Pathogenesis of non-insulin-dependent diabetes mellitus. Lancet 1994, 34:91-95.

45. Weijers RNM, Bekedam DJ: Relationship between gestational diabetes mellitus and type 2 diabetes. Clin Chem 2007, 53:377-383.

46. Chausmer AB: Zinc, insulin and diabetes [review]. J Am Coll Nutr 1998 17:109-115

doi: $10.1186 / 1758-5996-2-33$

Cite this article as: Weijers, Three-dimensional structure of ?-cell-specific zinc transporter, ZnT-8, predicted from the type 2 diabetes-associated gene variant SLC30A8 R325W Diabetology \& Metabolic Syndrome 2010, 2:33 